\title{
Version of Accelerating Expansion Is in Contradistinction to a Lot of Facts of Observations
}

\author{
Banggu Zhang, Bangning Zhang, Yinan Zhang \\ China Academy of Space Technology, Beijing, China \\ Email: bangguzhang@163.com
}

How to cite this paper: Zhang, B.G., Zhang, B.N. and Zhang, Y.N. (2017) Version of Accelerating Expansion Is in Contradistinction to a Lot of Facts of Observations. Open Access Library Journal, 4: e4035. https://doi.org/10.4236/oalib.1104035

Received: October 16, 2017

Accepted: November 12, 2017

Published: November 15, 2017

Copyright $\odot 2017$ by authors and Open Access Library Inc.

This work is licensed under the Creative Commons Attribution International License (CC BY 4.0).

http://creativecommons.org/licenses/by/4.0/

\section{(c) (i) Open Access}

\begin{abstract}
The term "accelerating expansion" is contradicted to follow observation facts: Accelerating expansion means repulsive force account for $70 \%$ and the universal space will be open. However, the observation facts indicate that the cosmetic space is flat and cosmos microwave background radiation is blackbody radiation. That is to say, it is equilibrium radiation. The cosmos is a gravitationally bound system. Obviously, open space cannot form bound system. A lot of observation facts and the Hubble relation support that the cosmos is always in reduction expansion. The term is also contradicted to the warped and closed space demanded by cosmetic principle.
\end{abstract}

\section{Subject Areas}

Applied Physics

\section{Keywords}

Standard Model, Supernova, Flat Space, Background Radiation, Hubble Relation, Decelerating Expansion

\section{Introduction}

In the beginning of this century, it is said scientists in Australia and the US discovered a super nova 26 billion light years away with red shift over 10. Obviously, it is a discovery which breaks two world records and should be given Nobel Prize in physics. In addition, the existence of this 26 billion light years away super nova breaks the concept that the age of the cosmos is about a hundred billion years in the "standard model" of cosmology. Its theoretical significance is obvious.

However, the Nobel Prize in physics was awarded to "the discovery of the ac- 
celerating expansion".

We think, "accelerating expansion" is not coincide with the reality [1] [2]. Dr. Xiaochun Mei published an article in which said, this is a mistake by the Noble Prize committee.

Recently, we had some discussions and though we should have some further discussion on this in manuscript.

\section{Result and Discussion:}

\section{1) The average density of the Universe}

According to the standard model [3], if the average density of the Universe is larger than the critical density, the space will be warped and closed (spherical surface in two-dimensional space). This is because the higher the density, the higher the gravity will be which will make the space warped and closed. If the density is equal to the critical density, the space will be flat (flat surface in twodimensional space). If the density is smaller than the critical density, the space will be open (double curved surface in two-dimensional space) because of small gravity.

From many measurements, including dark substance, the average density of the Universe is only $30 \%$ of the critical density.

That is to say, the theory of the standard model is not coincide with reality.

\section{2) Accelerating expansion, dark energy}

A new observed result seemed to give hope to the standard model. Some super nova seem too dark. Therefore, some scientists explained that they are too far away and they can only be reached by accelerating expansion. Accelerating expansion is made by "dark energy" with repulsive force. Thus, with "dark energy" (about $70 \%$ of the critical density), the average density of the Universe can get the critical density, therefore coincide with observed facts.

\section{3) Strange logic}

According to the standard model, the critical factor that decides the properties of space is gravity. If the gravity is large, space will be warped and closed; if the gravity is not big enough, the space will be flat; if the gravity is small, the space will be open.

Without "dark energy", the density of the Universe is small, the gravity is small, thus the space should be open. With repulsive "dark energy", the gravity should be even smaller, the space should be more open. How can it be coincide with the observed fact that the space is flat?

\section{4) Contradict with background radiation}

As we know, cosmetic microwave background radiation [4] is a blackbody radiation. It is a photon equilibrium state in fact [5]. That is to say, in any point in the space (such as the earth), there will be photons from every direction.

If repulsive force account for $70 \%$, the universal space will be open [3]. Then, the photons produced at the big bang will be repelled to faraway. How can they be bounded to form equilibrium state? 
Open space implies there should be a center of mass in the Universe. It is the point which big bang occurred. If repulsive force is the major, then, how could be so many photons move toward the center of mass?

\section{5) Contradict with Hubble relation}

In 1929, Hubble analyzed observed data of 46 galaxies and found, their velocity of recession $v$ is direct proportional to their distance $r$. That's to say, in a coordinate system with velocity as the vertical axis and distance as the transverse axis, this function is an upward line. The maximum red shift is 0.004 at that time. In the following 20 years, Hubble, Humason, Mayall analyzed 850 galaxies with the maximum red shift about $1 / 3$. All of them coincide with Hubble relation [6].

Hubble relation [7] states the velocity of recession of galaxies is equal to

$$
v=H r
$$

That is to say, the farther the galaxies, the bigger the velocity of recession will be. The $H$ is called Hubble constant.

As we know, the velocity and distance of galaxies were got from the observation of their light. These lights were emitted from

$$
t=r / c
$$

before.

For example, the light observed from a galaxy 1 million light years away were emitted 1 million years before. That is to say, the velocity got from red shift is the velocity of the galaxy 1 million years before.

Recession of galaxies reflect the expansion of the cosmos essentially. Thus, the earlier the time, the bigger the expansion velocity will be. That is to say, expansion of the Universe is decelerating. From above two equations, it is easy to get the deceleration is

$$
\mathrm{d} v / \mathrm{d} t=H c
$$

For decades, with more observed data, the red shift is bigger and bigger. As it is over $1 / 3$, the function is no longer linear [8]. As the velocity is close to the light velocity, corresponding equation in relativity should be used to calculate velocity from observed red shift. If consider this red shift is mainly created by Doppler effect, then, by corresponding equation in relativity [9], following results could be got: a celestial body with red shift of one will move away from us with speed of $60 \%$ light speed, a celestial body with red shift of three will have a speed of $90 \%$ light velocity, a celestial body with red shift of six will have a speed of $96 \%$ light velocity. Figure 1 demonstrates the function is a curve approaching line $v=c$. However, the curve is still upward. Velocity is still direct proportion to distance.

This is the reason Hubble law changed to Hubble relation [10].

Since $1920^{\text {th }}$ Hubble relation was proposed, many observations of recession of galaxies coincided with Hubble relation that recession speed direct proportion to distance. That is to say, many observed facts indicate that expansion of cosmos is 


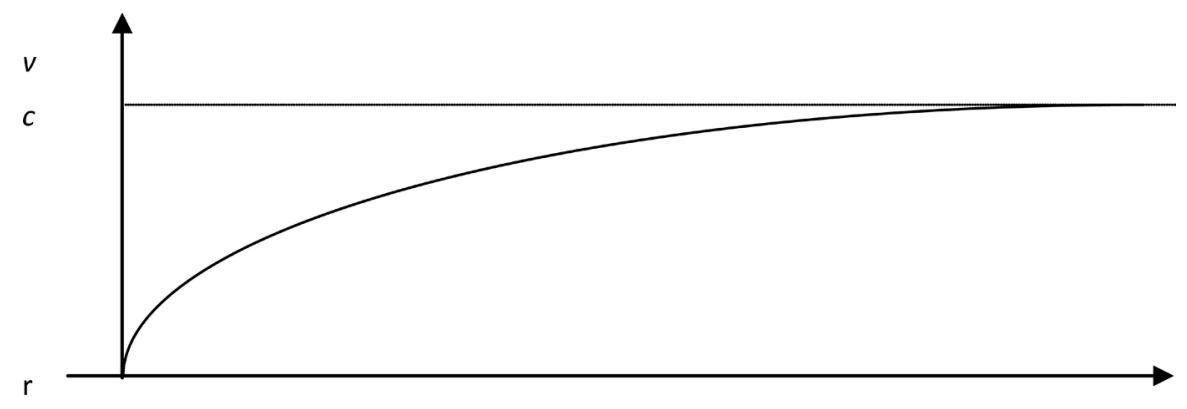

Figure 1. The relationship of recession speed with distanceof galaxies.

always decelerating. There is no doubt that "accelerating expansion" cannot exist.

6) More and more trouble

Old question is not solved and another bigger problem appear: what is the repulsive "dark energy"? The proposer didnot state clearly.

"Accelerating expansion" is not a direct result from observation.

From the first manuscript that proposed accelerating expansion published at the end of last century, we did not find any data from direct measurement. In fact, the author put observed data into the standard model and after some analysis got the result of accelerating expansion. Furthermore, for seeking the force of accelerating expansion, he proposed the concept of dark energy.

Here, we hope the author can publish the origin data of the brightness of the observed star and the distance calculated from itand red shift, so more people can share his results. And we hope other people done similar observational experiments can open access the data as well.

\section{Conclusion}

The term "accelerating expansion" is contradicted to lots of observation facts, it is not a direct result from observations.

\section{References}

[1] Zhang, B.G. (2014) Navigation in the Cosmos. World Culture Publishing House, 56. (In Chinese)

[2] Zhang, B.G. (2015) Mystery of the Cosmos. 2nd Edition, Scientific Research Publishing, 64.

[3] Weinberg, S. (1972) Gravitation and Cosmology. John Wiley, Chapter 15.

[4] Weinberg, S. (1972) Gravitation and Cosmology. John Wiley, Section 15.5.

[5] Zhang, B.G. (1998) Background Radiation and Photon Equilibrium State. Journal of Light Scattering, 10, 47.

[6] Yu, Y.Q. (2002) Lectures on Physicalcosmology. Beijing University Press, Beijing, 74. (In Chinese)

[7] Yu, Y.Q. (1998) Introduction to General Relativity. Beijing University Press, Beijing, 144. (In Chinese)

[8] Zhang, B.G. (2015) Theoretical Astrophysics. Scientific Research Publishing, 31.

[9] Zhang, B.G. (2015) Mystery of the Space. Scientific Research Publishing, 158.

[10] Weinberg, S. (1972) Gravitation and Cosmology. John Wiley, Section 14.1. 
Submit or recommend next manuscript to OALib Journal and we will provide best service for you:

- Publication frequency: Monthly

- 9 subject areas of science, technology and medicine

- Fair and rigorous peer-review system

- Fast publication process

- Article promotion in various social networking sites (LinkedIn, Facebook, Twitter, etc.)

- Maximum dissemination of your research work

Submit Your Paper Online: Click Here to Submit

Or Contact service@oalib.com 